TRANSACTIONS OF THE

AMERICAN MATHEMATICAL SOCIETY

Volume 350, Number 4, April 1998, Pages 1595-1618

S 0002-9947(98)02051-0

\title{
COMMUTING TOEPLITZ OPERATORS WITH PLURIHARMONIC SYMBOLS
}

\author{
DECHAO ZHENG
}

\begin{abstract}
By making use of $\mathcal{M}$-harmonic function theory, we characterize commuting Toeplitz operators with bounded pluriharmonic symbols on the Bergman space of the unit ball or on the Hardy space of the unit sphere in $n$-dimensional complex space.
\end{abstract}

\section{INTRODUCTION}

Let $d A(z)$ denote the Lebesgue volume measure on the open unit ball $B_{n}$ of $C^{n}$, normalized so that the measure of $B_{n}$ equals 1 . The Bergman space $L_{a}^{2}\left(B_{n}\right)$ is the Hilbert space consisting of holomorphic functions on $B_{n}$ that are also in $L^{2}\left(B_{n}, d A\right)$.

For $f$ in $L^{\infty}\left(B_{n}\right)$, the Toeplitz operator $T_{f}$ with symbol $f$ is defined by $T_{f}(h)=$ $P(f h)$, where $P$ is the orthogonal projection from $L^{2}\left(B_{n}, d A\right)$ onto $L_{a}^{2}\left(B_{n}, d A\right)$, called the Bergman projection.

Let $d \sigma$ be the surface area measure on the unit sphere $S_{n}$. For $1 \leq p<\infty$, $H^{p}\left(S_{n}\right)$ is the Banach space of holomorphic functions on $B_{n}$ with the norm defined by

$$
\|f\|_{p}=\operatorname{Sup}\left\{\left.\left.\left|\int_{S_{n}}\right| f(r z)\right|^{p} d \sigma(z)\right|^{1 / p} ; 0<r<1\right\} .
$$

For $f$ in $L^{\infty}\left(S_{n}, d \sigma\right)$, the Toeplitz operator $T_{f}$ with symbol $f$ is defined by $T_{f}(h)=S(f h)$, where $S$ is the orthogonal projection from $L^{2}\left(S_{n}, d \sigma\right)$ onto the Hardy space $H^{2}\left(S_{n}\right)$, called the Hardy projection. Here we use the same notation $T_{f}$ to denote the Toeplitz operator on both $L_{a}^{2}\left(B_{n}\right)$ and $H^{2}\left(S_{n}\right)$.

In this paper we study commuting Toeplitz operators on the Bergman space $L^{2}\left(B_{n}\right)$ of the unit ball $B_{n}$ or on the Hardy space $H^{2}\left(S_{n}\right)$ of the unit sphere $S_{n}$, using harmonic function theory on the unit ball $B_{n}$.

In the study of one complex variable, a real valued function $f$ on the unit disk is harmonic if $\Delta f=0$, where

$$
\Delta=4 \frac{\partial^{2}}{\partial z \partial \bar{z}}
$$

is the Laplacian in $C$. It is well-known that $f$ is harmonic in $B_{1}$ if and only if $f$ is the real part of a holomorphic function.

Received by the editors June 30, 1995 and, in revised form, July 15, 1996.

1991 Mathematics Subject Classification. Primary 47B35.

Key words and phrases. Toeplitz operator, Bergman space, Hardy space, pluriharmonic function, $\mathcal{M}$-harmonic function.

Supported in part by the National Science Foundation.

(C)1998 American Mathematical Society 
In addition to the usual Laplacian on $B_{1}$, there is also the Laplace-Beltrami operator $\tilde{\Delta}$ which is defined in terms of the Bergman kernel function of $B_{1}$. This operator is given by

$$
\tilde{\Delta}=2\left(1-|z|^{2}\right)^{2} \frac{\partial^{2}}{\partial z \partial \bar{z}} .
$$

A real valued function $f$ is $\mathcal{M}$-harmonic if $\tilde{\Delta} f=0$. It is clear that $\tilde{\Delta} f=0$ if and only if $\Delta f=0$. So $f$ is harmonic if and only if $f$ is $\mathcal{M}$-harmonic.

When one considers $B_{n}, n>1$, there are three concepts of "harmonic", namely harmonic, $\mathcal{M}$-harmonic and pluriharmonic, and as a general rule, these are all different. The three definitions are as follows:

(1) $f$ is harmonic in $B_{n}$ if $\Delta f=0$ in $B_{n}$, where

$$
\Delta=4 \sum_{j=1}^{n} \frac{\partial^{2}}{\partial z_{j} \partial \overline{z_{j}}}
$$

is the usual Laplacian induced by the Euclidean metric on $B_{n}$.

(2) $f$ is pluriharmonic if

$$
\frac{\partial^{2} f}{\partial z_{i} \partial \overline{z_{j}}}=0
$$

for all $i, j$.

(3) $f$ is $\mathcal{M}$-harmonic if

$$
\tilde{\Delta} f=0
$$

where $\tilde{\Delta}$ is the Laplace-Beltrami operator induced by the Bergman metric on $B_{n}$.

A pluriharmonic function in the unit ball is the sum of a holomorphic function and the conjugate of a holomorphic function. But the mean value property does not completely characterize pluriharmonic functions. Instead it completely characterizes $\mathcal{M}$-harmonic functions. So function theory is complicated and rich on the unit ball.

Although in this paper we are concerned only with Toeplitz operators with pluriharmonic symbols, we will need to study $\mathcal{M}$-harmonic functions. By making use of $\mathcal{M}$-harmonic function theory, we completely characterize commuting Toeplitz operators with bounded pluriharmonic symbols either on the Bergman space $L_{a}^{2}\left(B_{n}\right)$ or on the Hardy space $H^{2}\left(S_{n}\right)$.

This paper is arranged as follows. Section 2 contains statements of the main results. Section 3 contains preliminaries on function theory on $B_{n}$. Section 4 contains lemmas which will be used in Section 5 . Section 5 contains results on $\mathcal{M}$ harmonic functions, which are the key in the proof of the main results. Section 6 contains proofs of our main results.

The author would like to thank S. Axler for pointing out Theorem 2.2, and he is grateful to J. Pincus for encouragement.

\section{MAIn Results}

We state our main results in this section. We postpone the proofs of these theorems until Section 6 . 
Theorem 2.1. Suppose that $\varphi$ and $\psi$ are bounded pluriharmonic functions on the unit ball. Then

$$
T_{\varphi} T_{\psi}=T_{\psi} T_{\varphi}
$$

on the Bergman space $L_{a}^{2}\left(B_{n}\right)$ if and only if $\varphi$ and $\psi$ satisfy one of the following conditions:

(1) Both $\varphi$ and $\psi$ are holomorphic on $B_{n}$.

(2) Both $\bar{\varphi}$ and $\bar{\psi}$ are holomorphic on $B_{n}$.

(3) Either $\varphi$ or $\psi$ is constant on $B_{n}$.

(4) There is a nonzero constant b such that $\varphi-b \psi$ is constant on $B_{n}$.

A special case of Theorem 2.1 on the unit disk was first proved by Axler and Gorkin $[\mathrm{AxG}]$, and the author [Zh1]. Axler and Čučković proved Theorem 2.1 in the case that $n=1$. Some partial results on commuting Toeplitz operators were obtained on the Bergman spaces of the unit ball in [CL] and [Zh2].

Theorem 2.1 does not hold if "pluriharmonic" is replaced by "measurable" or even "continuous". For example, if $\varphi$ and $\psi$ are bounded measurable radial functions (meaning $\varphi(z)=\varphi(\|z\|)$ and $\psi(z)=\psi(\|z\|)$ for all $z \in B_{n}$ ) on the unit ball $B_{n}$, then $T_{\varphi} T_{\psi}=T_{\psi} T_{\varphi}$. This example is based on a example on the unit disk in $[\mathrm{AxC}]$.

Theorem 2.2. Suppose that $\varphi$ and $\psi$ are bounded pluriharmonic functions on the unit ball. Then

$$
T_{\varphi} T_{\psi}=T_{\psi} T_{\varphi}
$$

on the Hardy space $H^{2}\left(S_{n}\right)$ if and only if $\varphi$ and $\psi$ satisfy one of the following conditions:

(1) Both $\varphi$ and $\psi$ are holomorphic on $B_{n}$.

(2) Both $\bar{\varphi}$ and $\bar{\psi}$ are holomorphic on $B_{n}$.

(3) Either $\varphi$ or $\psi$ is constant on $B_{n}$.

(4) There is a nonzero constant $b$ such that $\varphi-b \psi$ is constant on $B_{n}$.

Brown and Halmos $[\mathrm{BrH}]$ proved Theorem 2.2 when $n=1$. They examined the matrix products of Toeplitz operators on the Hardy space. On the Bergman space or the Hardy space $H^{2}\left(S_{n}\right), n>1$, Toeplitz operators do not have nice matrix representations. Thus function theory plays a large role in this paper.

The difficult part of the proof of Theorems 2.1 and 2.2 is to show that the conditions in these theorems are necessary for $T_{\varphi} T_{\psi}=T_{\psi} T_{\varphi}$. We reduce it to the following question about $\mathcal{M}$-harmonic functions:

Question 2.3. If $f, g, h$, and $k$ are holomorphic functions in $B_{n}$, when is $f \bar{g}-h \bar{k}$ $\mathcal{M}$-harmonic?

This question is very subtle. For a pair of functions $f$ and $g$ (that is, $h=k=0$ ), Ahern and Rudin $[\mathrm{AhR}]$ answered the question completely. And in [Zh2] a different method was used to study the question for a pair of functions. In this paper, we apply the same method as in [Zh2] to answer Question 2.3 completely when these functions are in $H^{2 n}\left(S_{n}\right)$.

\section{PReliminaries ON FUnCtion theORY}

This section contains results on the function theory of the unit ball, which will be needed later. A good reference for this material is Rudin's book [Rud]. 
For $z$ in $B_{n}$, let $\varphi_{z}$ be the Möbius transformation from $B_{n}$ onto $B_{n}$ defined by

$$
\varphi_{z}(w)=\frac{z-P_{z} w-\left(1-\|z\|^{2}\right)^{1 / 2} Q_{z} w}{1-(w, z)},
$$

where $P_{z}$ is the orthogonal projection from $C^{n}$ onto the subspace of $C^{n}$ spanned by $z$, and $Q_{z}=I-P_{z} \cdot \varphi_{z}$ is in the group $\operatorname{Aut}\left(B_{n}\right)$ of all biholomorphic maps of $B_{n}$ onto $B_{n}$. In fact, each $\varphi \in \operatorname{Aut}\left(B_{n}\right)$ has a unique representation $\varphi=U \circ \varphi_{z}$ for some $z \in B_{n}$ and some $n \times n$ unitary matrix $U$.

For any $z$ in $B_{n}$, the pointwise evaluation of functions in $L_{a}^{2}\left(B_{n}\right)$ at $z$ is a bounded functional. So there is a function $K_{z}$ in $L_{a}^{2}\left(B_{n}\right)$ such that

$$
f(z)=\left(f, K_{z}\right)
$$

for all $f$ in $L_{a}^{2}\left(B_{n}\right) . K_{z}$ is called the Bergman reproducing kernel. Indeed, we have

$$
K_{z}(w)=\frac{1}{(1-(w, z))^{n+1}} .
$$

For convenience we let $k_{z}$ denote the normalized Bergman kernel $\frac{K_{z}(w)}{K_{z}(z)}$. As $K_{z}(z)>$ 0 for any $z$ in $B_{n}, B_{n}$ admits an Hermitian metric, the so-called Bergman metric, given on $B_{n}$ by

$$
g_{i j}(z)=\frac{\partial^{2}}{\partial z_{i} \partial \bar{z}_{j}} \log K_{z}(z)
$$

for $z$ in $B_{n}$. The Laplace-Beltrami operator for the Bergman metric of $B_{n}$ is given by

$$
\tilde{\Delta}=c_{n}\left(1-\|z\|^{2}\right)\left[\sum_{k} \frac{\partial^{2}}{\partial z_{k} \partial \bar{z}_{k}}-\sum_{i j} z_{i} \bar{z}_{j} \frac{\partial^{2}}{\partial z_{i} \partial \bar{z}_{j}}\right]
$$

for some positive constant $c_{n}$.

For any $f$ in $C^{2}\left(B_{n}\right)$, the gradient of $f$ is given by

$$
\nabla f(w)=\left(\frac{\partial f}{\partial z_{1}}(w), \cdots, \frac{\partial f}{\partial z_{n}}(w)\right)^{T} .
$$
if

A calculation in [Rud] shows that a function $u$ is $\mathcal{M}$-harmonic on $B_{n}$ if and only

$$
\sum_{i=1}^{n}\left(\frac{\partial}{\partial z_{i}} \frac{\partial}{\partial \overline{z_{i}}} u\right)(z)=\sum_{i, j=1}^{n} z_{i} \overline{z_{j}}\left(\frac{\partial}{\partial z_{i}} \frac{\partial}{\partial \overline{z_{j}}} u\right)(z)
$$

for all $z \in B_{n}$. It is clear that all holomorphic functions on $B_{n}$ are $\mathcal{M}$-harmonic, as are the pluriharmonic ones. Since each $\varphi \in \operatorname{Aut}\left(B_{n}\right)$ is an isometry of $B_{n}$ in the Bergman metric, the space of all $\mathcal{M}$-harmonic functions is invariant under the action of $\operatorname{Aut}\left(B_{n}\right)$.

The bounded $\mathcal{M}$-harmonic functions are characterized by the so-called invariant volume mean value property, i.e., a function $f$ bounded on $B_{n}$ is $\mathcal{M}$-harmonic if and only if

$$
f(\psi(0))=\int_{B} f \circ \psi d V
$$

for every $\psi \in \operatorname{Aut}\left(B_{n}\right)$ ([AFR], [Eng]). 
Let $\mathcal{U}$ be the group of all $n \times n$ unitary matrices. Clearly, $\mathcal{U}$ is a compact group. It is well-known that there is a Haar measure $d \mathcal{U}$ on $\mathcal{U}$. If $f$ is a measurable function on $B_{n}$, the identity

$$
\int_{S} f d \sigma=\int_{\mathcal{U}} f(\mathcal{U} \eta) d \mathcal{U}
$$

holds for any $\eta \in S_{n}$.

To identify a holomorphic function in the Hardy space $H^{2}\left(S_{n}\right)$, we need the following formula:

$$
\int_{S_{n}}\left|(\eta)^{\alpha}\right|^{2} d \sigma(\eta)=\frac{(n-1) ! \alpha !}{(n-1+|\alpha|) !}
$$

for every multi-index $\alpha$.

\section{Some Lemmas}

In this section we prove several lemmas which will be used in Section 5. First we need to introduce some concepts and notations.

Let $H\left(B_{n}\right)$ denote the space of holomorphic functions in $B_{n} . H\left(B_{n}\right)$ is a linear space. We say that functions $\left\{f_{i}\right\}_{i=1}^{n} \subset H\left(B_{n}\right)$ are linearly independent if there is no nonzero vector $\left(c_{1}, \cdots, c_{n}\right)^{T} \in C^{n}$ such that $\sum_{i=1}^{n} c_{i} f_{i}(z)=0$ for all $z \in B_{n}$.

For a vector $c=\left(c_{1}, \cdots, c_{n}\right)^{T} \in C^{n}$ and $f \in H\left(B_{n}\right)$, define

$$
\frac{\partial f}{\partial c}=\sum_{i=1}^{n} \overline{c_{i}} \frac{\partial f}{\partial z_{i}}
$$

A polynomial $p(z)$ in $C^{n}$ is said to be homogeneous of degree $s$ if $p(t z)=t^{s} p(z)$. If $f(z)$ is holomorphic in a neighborhood of the origin in $C^{n}$, then the power series of $f$ can be written in the grouped form

$$
f(z)=\sum_{s=0}^{\infty} f_{s}(z)
$$

where $f_{s}(z)$ is a homogeneous polynomial of degree $s$. Equation (4.1) is called the homogeneous expansion of $f$. By Euler's theorem,

$$
\sum_{i=1}^{n} z_{i} \frac{\partial f_{s}}{\partial z}(z)=s f_{s}(z)
$$

We will often use this fact in this and the next section.

Lemma 4.2. For any $f \in H\left(B_{n}\right)$, there is an $n \times n$ unitary matrix $U$ such that for some $1 \leq k \leq n$, the functions $\frac{\partial(f \circ U)}{\partial z_{1}}, \cdots, \frac{\partial(f \circ U)}{\partial z_{k}}$ are linearly independent and $\frac{\partial(f \circ U)}{\partial z_{i}}=0$ for all $i>k$.

Proof. Let $\mathcal{Z}=\left\{c \in C^{n}: \frac{\partial f}{\partial c}=0\right\}$. $\mathcal{Z}$ is a subspace of $C^{n}$. Then there is an orthonormal basis $\left\{e_{1}, \cdots, e_{n}\right\}$ of $C^{n}$ such that for some $1 \leq k \leq n,\left\{e_{k+1}, \cdots, e_{n}\right\}$ is also an orthonormal basis of $Z$. Let $U=\overline{\left(e_{1}, \cdots, e_{n}\right)}$. Then $U$ is a unitary matrix. On the other hand, by the chain rule, letting $w=U z$, we have

$$
\frac{\partial(f \circ U)}{\partial z_{i}}=\frac{\partial f}{\partial e_{i}}(w)
$$


Note that for $i>k, e_{i}$ is in $\mathcal{Z}$, and so

$$
\frac{\partial(f \circ U)}{\partial z_{i}}=0
$$

for all $i>k$.

To complete the proof of this lemma, we need to show that $\frac{\partial(f \circ U)}{\partial z_{1}}, \cdots, \frac{\partial(f \circ U)}{\partial z_{k}}$ are linearly independent. Suppose that there are constants $c_{i}$ so that

$$
\sum_{i=1}^{k} c_{i} \frac{\partial(f \circ U)}{\partial z_{i}}=0
$$

Then by the chain rule again, we obtain

$$
\sum_{i=1}^{k} c_{i} e_{i}^{*}(\nabla f)(U z)=0,
$$

and so $\sum_{i=1}^{k} \overline{c_{i}} e_{i} \in \mathcal{Z}$. But $e_{i}$ is orthogonal to $\mathcal{Z}$ for $i=1, \cdots, k$. Thus we conclude that $c_{i}=0$ for $i=1, \cdots, k$, completing the proof.

Lemma 4.3. Suppose that $c=\left(c_{1}, \cdots, c_{n}\right)$ is in the closure of $B_{n}$. Then the equation

$$
\sum_{i=1}^{n} z_{i} \frac{\partial f}{\partial z_{i}}=\sum_{i=1}^{n} c_{i} \frac{\partial f}{\partial z_{i}}
$$

does not have any non-constant solutions in $H^{2 n}\left(S_{n}\right)$.

Proof. Suppose that Equation (4.4) has a solution $f$ in $H^{2 n}\left(S_{n}\right)$. We consider two cases, $c \in B_{n}$ and $c \in S_{n}$. In each case we will show that $f$ is constant.

Case 1. In this case assume $c$ is in $B_{n}$. Making the change of variables $w=z-c$, and using the chain rule, we have

$$
\sum_{i=1}^{n} w_{i} \frac{\partial f}{\partial w_{i}}(w+c)=0 .
$$

Let $g(w)=f(w+c)$; then the above equation becomes

$$
\sum_{i=1}^{n} w_{i} \frac{\partial g}{\partial w_{i}}(w)=0
$$

Since $f$ is holomorphic on the open neighborhood $B_{n}$ of $c, g$ is holomorphic on the neighborhood $B_{n}-c$ of 0 and satisfies

$$
\sum_{i=1}^{n} w_{i} \frac{\partial g}{\partial w_{i}}(w)=0
$$

for all $w \in B_{n}-c$. Clearly, the above equation holds on $r B_{n} \subset B_{n}-c$ for some $0<r<1$. Applying Euler's theorem to the homogeneous expansion of $g$ yields

$$
\sum_{s=0}^{\infty} s g_{s}(w)=0
$$

for $w$ in $r B_{n}$. Then $g_{s}(w)=0$ for $s>0$, and so $g$ is constant on $r B_{n}$. Thus we conclude that $f$ is constant on $B_{n}$. 
Case 2. Now we assume $c$ is in $S_{n},\|c\|=1$. It follows from linear algebra that there is an $n \times n$ unitary matrix $U$ such that

$$
c^{T}(U)^{T}=(\|c\|, 0, \cdots, 0) .
$$

Use the chain rule again to get

$$
(U z)^{T} \nabla\left(f \circ U^{*}\right)(U z)=z^{T} \nabla f(z)
$$

and

$$
\begin{gathered}
\|c\| \frac{\partial\left(f \circ U^{*}\right)}{\partial z_{1}}(U z)=(\|c\|, 0, \cdots, 0) \nabla\left(f \circ U^{*}\right)(U z) \\
=(\|c\|, 0, \cdots, 0)\left(U^{*}\right)^{T} \nabla f(z)=c^{T} \nabla f(z) .
\end{gathered}
$$

Let $g=f \circ U^{*}$. Clearly, $g$ is in $H^{2 n}\left(S_{n}\right)$. Combining Equation (4.4) with the above equations gives

$$
\sum_{i=1}^{n} z_{i} \frac{\partial g}{\partial z_{i}}=\frac{\partial g}{\partial z_{1}}
$$

Write $g$ as a homogeneous expansion

$$
g(z)=\sum_{t=0}^{\infty} \sum_{s=0}^{\infty} g_{t, s}\left(z_{2}, \cdots, z_{n}\right) z_{1}^{t},
$$

where $g_{t, s}\left(z_{2}, \cdots, z_{n}\right)$ is homogeneous of degree $s$ in $z_{2}, \cdots, z_{n}$. Putting the above homogeneous expansion into Equation (4.5) and then applying Euler's theorem, we get

$$
\sum_{t=0}^{\infty} \sum_{s=0}^{\infty}(s+t) g_{t, s}\left(z_{2}, \cdots, z_{n}\right) z_{1}^{t}=\sum_{t=0}^{\infty} \sum_{s=0}^{\infty} t g_{t, s}\left(z_{2}, \cdots, z_{n}\right) z_{1}^{t-1} .
$$

Comparing the coefficients of powers of $z_{1}$ in the above equation gives

$$
(s+t) g_{t, s}\left(z_{2}, \cdots, z_{n}\right)=(t+1) g_{t+1, s}\left(z_{2}, \cdots, z_{n}\right) .
$$

This implies the following reduction formula for $t$ :

$$
g_{t+1, s}\left(z_{2}, \cdots, z_{n}\right)=\frac{s+t}{t+1} g_{t, s}\left(z_{2}, \cdots, z_{n}\right) .
$$

Iterating the formula gives

$$
g_{t, s}\left(z_{2}, \cdots, z_{n}\right)=\frac{(s+t-1) !}{t ! s !} g_{0, s}\left(z_{2}, \cdots, z_{n}\right) .
$$

Thus $g$ is expressed as

$$
\begin{gathered}
g(z)=\sum_{t=0}^{\infty} \sum_{s=0}^{\infty} \frac{(s+t-1) !}{t ! s !} g_{0, s}\left(z_{2}, \cdots, z_{n}\right) z_{1}^{t} \\
=\sum_{s=0}^{\infty}\left(\sum_{t=0}^{\infty} \frac{(s+t-1) !}{t ! s !} z_{1}^{t}\right) g_{0, s}\left(z_{2}, \cdots, z_{n}\right) \\
=\sum_{s=0}^{\infty} \frac{g_{0, s}\left(z_{2}, \cdots, z_{n}\right)}{\left(1-z_{1}\right)^{s}} .
\end{gathered}
$$


For the sake of simplicity, let $g_{s}$ be $g_{0, s}$. Noting that $g_{s}\left(z_{2}, \cdots, z_{n}\right)$ is a homogeneous polynomial of degree $s$, write it as

$$
g_{s}\left(z_{2}, \cdots, z_{n}\right)=\sum_{|\alpha|=s} a_{\alpha s} z^{(0, \alpha)} .
$$

So it is easy to verify that

$$
\|f\|_{2}^{2}=\sum_{s=0}^{\infty} \sum_{|\alpha|=s} \sum_{t=0}^{\infty}\left(\frac{(s+t-1) !}{t ! s !}\right)^{2}\left|a_{\alpha s}\right|^{2}\left\|z^{(t, \alpha)}\right\|_{2}^{2} .
$$

The formula (3.3) gives

$$
\|g\|_{2}^{2}=\sum_{s=0}^{\infty} \sum_{|\alpha|=s} \sum_{t=0}^{\infty}\left(\frac{(s+t-1) !}{t ! s !}\right)^{2} \frac{(n-1) ! \alpha ! t !}{(n-1+s+t) !}\left|a_{\alpha s}\right|^{2} .
$$

Since for $t$ very large,

$$
\left(\frac{(s+t-1) !}{t !}\right)^{2} \frac{\alpha ! t !}{(n-1+s+t) !}
$$

is asymptotically equivalent to

$$
\frac{1}{t^{n-s+1}}
$$

in order that the series (4.6) converge, $n-s+1$ must be greater than one. Therefore

$$
g(z)=\sum_{s \leq s_{0}} \frac{f_{s}\left(z_{2}, \cdots, z_{n}\right)}{\left(1-z_{1}\right)^{s}}
$$

for some $0 \leq s_{0}<n$. It is easy to see that the term with the highest degree of $\frac{1}{\left(1-z_{1}\right)}$ in $g^{n}$ is

$$
\frac{g_{s_{0}}^{n}\left(z_{2}, \cdots, z_{k}\right)}{\left(1-z_{1}\right)^{s_{0} n}}
$$

On the other hand, $g^{n}$ is in $H^{2}\left(S_{n}\right)$. Thus $s_{0} n<n$, and so $s_{0}=0$. This means that $g$ is constant. Noting that $g=f \circ U^{*}$, we get that $f$ is constant, completing the proof.

Lemma 4.7. For each $j$, the equation

$$
\frac{\partial}{\partial z_{j}}\left(\sum_{i=1}^{n} z_{i} \frac{\partial f}{\partial z_{i}}\right) \overline{\left(\sum_{i=1}^{n} w_{i} \frac{\partial g}{\partial w_{i}}\right)}=\frac{\partial}{\partial z_{j}} \sum_{i=1}^{n} \frac{\partial f}{\partial z_{i}} \overline{\frac{\partial g}{\partial w_{i}}}
$$

does not have a solution in $H^{2 n}\left(B_{n}\right)$ unless either $\frac{\partial f}{\partial z_{j}}$ is zero or $g$ is constant for any fixed $j$.

Proof. Assume that $g$ is not constant. Let $f$ be a solution in $H^{2 n}\left(S_{n}\right)$ of Equation (4.8). We will show that $\frac{\partial f}{\partial z_{j}}$ is constant. Since $g$ is not constant, for some $w \in B_{n}$,

$$
\sum_{i=1}^{n} w_{i} \frac{\partial g}{\partial w_{i}} \neq 0
$$


For a fixed $w$, solving for $\frac{\partial}{\partial z_{j}}\left(\sum_{i=1}^{n} z_{i} \frac{\partial f}{\partial z_{i}}\right)$ in (4.8) gives that for some vector $b \in C^{n}$,

$$
\frac{\partial}{\partial z_{j}}\left(\sum_{i=1}^{n} z_{i} \frac{\partial f}{\partial z_{i}}\right)=b^{*} \nabla \frac{\partial f}{\partial z_{j}} .
$$

Let $\mathcal{W}=\left\{c \in C^{n}: \frac{\partial^{2} f}{\partial c \partial z_{j}}=0\right\} . \mathcal{W}$ is a subspace of $C^{n}$. So there is a unique vector $a \in C^{n}$ such that

$$
\frac{\partial}{\partial z_{j}}\left(\sum_{i=1}^{n} z_{i} \frac{\partial f}{\partial z_{i}}\right)=a^{*} \nabla \frac{\partial f}{\partial z_{j}}
$$

where $a$ is orthogonal to $\mathcal{W}$. Putting (4.9) and (4.8) together gives

$$
a^{*} \nabla \frac{\partial f}{\partial z_{j}} \overline{\left(\sum_{i=1}^{n} w_{i} \frac{\partial g}{\partial w_{i}}\right)}=\nabla g(w)^{*} \nabla \frac{\partial f}{\partial z_{j}} .
$$

Thus

$$
a \sum_{i=1}^{n} w_{i} \frac{\partial g}{\partial w_{i}}-\nabla g(w)
$$

is in $\mathcal{W}$ for any $w$ in the unit ball $B_{n}$. Noting that $a$ is orthogonal to $\mathcal{W}$, we obtain

$$
\|a\|^{2} \sum_{i=1}^{n} w_{i} \frac{\partial g}{\partial w_{i}}-a^{*} \nabla g(w)=0 .
$$

By Lemma $4.3, \frac{a}{\|a\|^{2}}$ is not in the closure of $B_{n}$, and so $a$ is in $B_{n}$.

Now we write (4.9) as

$$
\frac{\partial}{\partial z_{j}} \sum_{i=1}^{n}\left(z_{i}-\overline{a_{i}}\right) \frac{\partial f}{\partial z_{i}}=0
$$

Making the change of variables $w=z-\bar{a}$ gives

$$
\frac{\partial}{\partial w_{j}} \sum_{i=1}^{n} w_{i} \frac{\partial f}{\partial w_{i}}(w+\bar{a})=0 .
$$

Let $F(w)=f(w+\bar{a})$; then the above equation becomes

$$
\frac{\partial}{\partial w_{j}} \sum_{i=1}^{n} w_{i} \frac{\partial F}{\partial w_{i}}(w)=0 .
$$

Since $f$ is holomorphic on the neighborhood $B_{n}$ of $\bar{a}$, for some $0<r<1, F$ is holomorphic on the neighborhood $r B_{n} \subset B_{n}-\bar{a}$ of 0 and satisfies

$$
\frac{\partial}{\partial w_{j}} \sum_{i=1}^{n} w_{i} \frac{\partial F}{\partial w_{i}}(w)=0
$$

on $r B_{n}$. Applying Euler's theorem to the homogeneous expansion of $F$ yields

$$
\frac{\partial}{\partial w_{j}} \sum_{s=0}^{\infty} s F_{s}(w)=0
$$

Thus $\frac{\partial F_{s}}{\partial w_{j}}(w)=0$ on $r B_{n}$ for $s>0$, and so $\frac{\partial F}{\partial w_{j}}(w)=0$. This implies that $\frac{\partial f}{\partial z_{j}}=0$, completing the proof. 


\section{5. $\mathcal{M}$-harmonic FUnCtions}

In this section we will characterize functions $f, g, h$, and $k$ in $H^{2 n}\left(S_{n}\right)$ such that $f \bar{g}-h \bar{k}$ is $\mathcal{M}$-harmonic. In [AhR], an explicit description is given of all pairs of holomorphic functions $f$ and $g$ in $B_{n}$ for which the product $f \bar{g}$ is $\mathcal{M}$-harmonic. Throughout this section we assume that $f, g, h$, and $k$ are holomorphic on the unit ball $B_{n}$.

Lemma 5.1. $f \bar{g}-h \bar{k}$ is $\mathcal{M}$-harmonic if and only if

$$
\begin{gathered}
\left(\sum_{i=1}^{n} z_{i} \frac{\partial f}{\partial z_{i}}\right) \overline{\left(\sum_{i=1}^{n} w_{i} \frac{\partial g}{\partial w_{i}}\right)}-\left(\sum_{i=1}^{n} z_{i} \frac{\partial h}{\partial z_{i}}\right) \overline{\left(\sum_{i=1}^{n} w_{i} \frac{\partial k}{\partial w_{i}}\right)} \\
=\left(\sum_{i=1}^{n} \frac{\partial f}{\partial z_{i}} \overline{\frac{\partial g}{\partial w_{i}}}-\sum_{i=1}^{n} \frac{\partial h}{\partial z_{i}} \overline{\frac{\partial k}{\partial w_{i}}}\right)
\end{gathered}
$$

for $z$ and $w$ in $B_{n}$.

Proof. As pointed out in Section 3, $u$ is $\mathcal{M}$-harmonic if and only if $u$ satisfies Equation (3.1). When $u=f \bar{g}-h \bar{k}$, then Equation (3.1) becomes

$$
\begin{gathered}
\left(\sum_{i=1}^{n} z_{i} \frac{\partial f}{\partial z_{i}}\right) \overline{\left(\sum_{i=1}^{n} z_{i} \frac{\partial g}{\partial z_{i}}\right)}-\left(\sum_{i=1}^{n} z_{i} \frac{\partial h}{\partial z_{i}}\right) \overline{\left(\sum_{i=1}^{n} z_{i} \frac{\partial k}{\partial z_{i}}\right)} \\
=\left(\sum_{i=1}^{n} \frac{\partial f}{\partial z_{i}} \overline{\frac{\partial g}{\partial z_{i}}}-\sum_{i=1}^{n} \frac{\partial h}{\partial z_{i}} \overline{\frac{\partial k}{\partial z_{i}}}\right) .
\end{gathered}
$$

Hence Equation (5.3) is a special case of (5.2) when $z=w$. So if $f, g, h$ and $k$ satisfy Equation (5.2), then $f \bar{g}-h \bar{k}$ is $\mathcal{M}$-harmonic.

Conversely, we will show that Equation (5.3) gives Equation (5.2). Let the homogeneous expansions of $f, g, h$, and $k$ be

$$
f(z)=\sum_{s=1}^{\infty} f_{s}(z), g(z)=\sum_{s=1}^{\infty} g_{s}(z)
$$

and

$$
h(z)=\sum_{s=1}^{\infty} h_{s}(z), k(z)=\sum_{s=1}^{\infty} k_{s}(z) .
$$

Euler's theorem implies that

$$
\sum_{i=1}^{n} z_{i} \frac{\partial f_{s}}{\partial z_{i}}=s f_{s}(z)
$$

So Equation (5.3) becomes

$$
\sum_{s, t=1}^{\infty} s t\left(f_{s}(z) \overline{g_{t}(z)}-h_{s}(z) \overline{k_{t}(z)}\right)=\sum_{s, t=0}^{\infty} \sum_{i=1}^{n}\left(\frac{\partial f_{s}}{\partial z_{i}} \overline{\frac{\partial g_{t}}{\partial z_{i}}}-\frac{\partial h_{s}}{\partial z_{i}} \overline{\frac{\partial k_{t}}{\partial z_{i}}}\right) .
$$


Let $z=\xi w$ for $\xi$ in the unit disk and $w \in B_{n}$. Note that $f_{s}, g_{s}, h_{s}$, and $k_{s}$ are homogeneous. An easy computation yields

$$
\begin{aligned}
& \sum_{s, t=1}^{\infty} s t \xi^{s} \overline{\xi^{t}}\left(f_{s}(w) \overline{g_{t}(w)}-h_{s}(w) \overline{k_{t}(w)}\right) \\
& =\sum_{s, t=0}^{\infty} \sum_{i=1}^{n} \xi^{s-1} \overline{\xi^{t-1}}\left(\frac{\partial f_{s}}{\partial w_{i}} \frac{\overline{\partial g_{t}}}{\partial w_{i}}-\frac{\partial h_{s}}{\partial w_{i}} \frac{\overline{\partial k_{t}}}{\partial w_{i}}\right) .
\end{aligned}
$$

Comparing the coefficients of $\xi^{s \overline{\xi^{t}}}$ in the above equation yields

$$
s t\left(f_{s}(w) \overline{g_{t}(w)}-h_{s}(w) \overline{\left.k_{t}(w)\right)}=\sum_{i=1}^{n}\left(\frac{\partial f_{s+1}}{\partial w_{i}} \frac{\overline{\partial g_{t+1}}}{\partial w_{i}}-\frac{\partial h_{s+1}}{\partial w_{i}} \frac{\overline{\partial k_{t+1}}}{\partial w_{i}}\right)\right.
$$

for any $s$ and $t$. Applying $\overline{D^{\alpha}}$ both sides of the above equation with respect to $w$ for $|\alpha|=t$ gives

$$
s t\left(f_{s}(w) \overline{D^{\alpha} g_{t}(w)}-h_{s}(w) \overline{D^{\alpha} k_{t}(w)}\right)=\sum_{i=1}^{n}\left(\frac{\partial f_{s+1}}{\partial w_{i}} \overline{D^{\alpha} \frac{\partial g_{t+1}}{\partial w_{i}}}-\frac{\partial h_{s+1}}{\partial w_{i}} \overline{D^{\alpha} \frac{\partial k_{t+1}}{\partial w_{i}}}\right) .
$$

But for any homogeneous polynomial $P$ of degree $t$

$$
P(w)=\sum_{|\alpha|=t} \frac{1}{\alpha !}\left[D^{\alpha} P\right] w^{\alpha} .
$$

Multiplying both sides of Equation (5.4) by $\frac{\bar{w}^{\alpha}}{\alpha !}$, and then taking the sum for $\alpha$ with $|\alpha|=t$, we get

$$
s t\left(f_{s}(w) \overline{g_{t}(z)}-h_{s}(w) \overline{k_{t}(z)}\right)=\sum_{i=1}^{n}\left(\frac{\partial f_{s+1}}{\partial w_{i}} \frac{\overline{\partial g_{t+1}}}{\partial z_{i}}-\frac{\partial h_{s+1}}{\partial w_{i}} \frac{\overline{\partial k_{t+1}}}{\partial z_{i}}\right) .
$$

Taking the sum with respect to $s$ and $t$ gives Equation (5.2) for any $z$ and $w$ in $B_{n}$, completing the proof.

Lemma 5.5. Suppose that neither $g$ nor $k$ is constant and $f \bar{g}-h \bar{k}$ is $\mathcal{M}$-harmonic. For any vector $a \in C^{n}$ and $a \neq 0$, if $\frac{\partial f}{\partial a}=0$, then $\frac{\partial h}{\partial a}=0$.

Proof. First we will show that $\frac{\partial f}{\partial z_{1}}=0$ implies $\frac{\partial h}{\partial z_{1}}=0$. Clearly, $\frac{\partial f}{\partial z_{1}}=0$ implies $\frac{\partial^{2} f}{\partial z_{1} \partial z_{j}}=0$ for all $j$. Differentiating both sides of Equation (5.2) with respect to $z_{1}$, we have

$$
\frac{\partial}{\partial z_{1}}\left(\sum_{i=1}^{n} z_{i} \frac{\partial h}{\partial z_{i}}\right) \overline{\left(\sum_{i=1}^{n} w_{i} \frac{\partial k}{\partial w_{i}}\right)}=\frac{\partial}{\partial z_{1}} \sum_{i=1}^{n} \frac{\partial h}{\partial z_{i}} \overline{\frac{\partial k}{\partial w_{i}}}
$$

Noting that $k$ is not constant, by Lemma 4.7 , we obtain $\frac{\partial h}{\partial z_{1}}=0$.

Next we reduce the general case to the above case. Assume that $\frac{\partial f}{\partial a}=0$ for some vector $a \neq 0$. For $e=(1,0, \cdots, 0)^{*} \in C^{n}$, let $U$ be a unitary matrix such that $\bar{U} e=a$. By the chain rule, we have

$$
\frac{\partial(f \circ U)}{\partial z_{1}}=\frac{\partial f}{\partial a}(U z) .
$$


On the other hand, the space of $\mathcal{M}$-harmonic functions is invariant under the action of unitary matrices. So $f \bar{g}-h \bar{k}$ implies $f \circ U \overline{g \circ U}-h \circ U \overline{k \circ U}$ is $\mathcal{M}$-harmonic. As shown above, $\frac{\partial(f \circ U)}{\partial z_{1}}=0$ implies $\frac{\partial(h \circ U)}{\partial z_{1}}=0$.

By the chain rule again, we have

$$
\frac{\partial(h \circ U)}{\partial z_{1}}=\frac{\partial h}{\partial a}(U z)
$$

which means $\frac{\partial h}{\partial a}=0$. This completes the proof.

If we assume that none of $f, g, h$ and $k$ is constant and $f \bar{g}-h \bar{k}$ is $\mathcal{M}$-harmonic, Lemma 5.5 tells us that any linear relation among derivatives of $f$ gives the same linear relation among derivatives of $h$. This fact is also valid for $g$ and $k$. We will often use this fact in the proof of Theorem 5.6. The following theorem is the main result in this section. It is the key to the proof of Theorems 2.1 and 2.2.

Theorem 5.6. Suppose that $f, g, h$, and $k$ are in $H^{2 n}\left(B_{n}\right)$. Then $f \bar{g}-h \bar{k}$ is $\mathcal{M}$ harmonic on the unit ball if and only if $f, g, h$, and $k$ satisfy one of the following conditions:

(1) Both $f$ and $h$ are constants.

(2) Both $f$ and $k$ are constants.

(3) Both $g$ and $k$ are constants.

(4) Both $g$ and $h$ are constants.

(5) There is a nonzero constant $b$ such that $b g-k$ and $f-\bar{b} h$ are constants.

Proof. If $f, g, h$ and $k$ satisfy one of the conditions (1)-(5) in this theorem, one easily sees that they satisfy Equation (5.2). Hence, by Lemma 5.1, $f \bar{g}-h \bar{k}$ is $\mathcal{M}$-harmonic.

Conversely, assume that $f \bar{g}-h \bar{k}$ is $\mathcal{M}$-harmonic. By Lemma 5.1, it is sufficient to show that any solutions in $H^{2 n}\left(B_{n}\right)$ of Equation (5.2) must satisfy one of the conditions in this theorem.

If one of $f, g, h$ and $k$ is constant, then (5.2) becomes an equation involving a pair of functions. In this case, it follows from a result in $[\mathrm{AhR}]$ and [Zh2] that one of the conditions (1)-(4) holds.

Now we may assume that none of $f, g, h$ and $k$ is constant. We will show that these functions satisfy condition (5) in this theorem. Note that Equation (5.2) is a linear equation in $\frac{\partial f}{\partial z_{1}}, \cdots, \frac{\partial f}{\partial z_{n}}, \sum_{i=1}^{n} z_{i} \frac{\partial f}{\partial z_{i}}, \frac{\partial k}{\partial z_{1}}, \cdots, \frac{\partial k}{\partial z_{n}}$, and $\sum_{i=1}^{n} z_{i} \frac{\partial k}{\partial z_{i}}$ for a fixed $w \in B_{n}$, and a linear equation in $\frac{\partial g}{\partial w_{1}}, \cdots, \frac{\partial g}{\partial w_{n}}, \sum_{i=1}^{n} w_{i} \frac{\partial g}{\partial w_{i}}, \frac{\partial h}{\partial w_{1}}, \cdots, \frac{\partial h}{\partial w_{n}}$, and $\sum_{i=1}^{n} w_{i} \frac{\partial h}{\partial w_{i}}$ for a fixed $z \in B_{n}$. We will use these equations to get condition (5).

First reduce the general case to a special case in which the derivatives of $f, g, h$ and $k$ satisfy the following conditions.

(a) $\frac{\partial f}{\partial z_{1}}, \cdots, \frac{\partial f}{\partial z_{r}}$ are linearly independent.

(b) $\frac{\partial g}{\partial w_{1}}, \cdots, \frac{\partial g}{\partial w_{r}}$ are linearly independent.

(c) $\frac{\partial h}{\partial z_{1}}, \cdots, \frac{\partial h}{\partial z_{r}}$ are linearly independent.

(d) $\frac{\partial k}{\partial w_{1}}, \cdots, \frac{\partial k}{\partial w_{r}}$ are linearly independent.

(e) Either $\frac{\partial f}{\partial z_{i}}=\frac{\partial h}{\partial z_{i}}=0$ or $\frac{\partial g}{\partial w_{i}}=\frac{\partial k}{\partial w_{i}}=0$ for $i>r>0$. 
By Lemma 4.2, there is a unitary matrix $V$ such that $\frac{\partial(f \circ V)}{\partial z_{1}}, \cdots, \frac{\partial(f \circ V)}{\partial z_{r_{1}}}$ are linearly independent and $\frac{\partial(f \circ V)}{\partial z_{i}}=0$ for $i>r_{1}$. By Lemma 5.5, $\frac{\partial(h \circ V)}{\partial z_{1}}, \cdots, \frac{\partial(h \circ V)}{\partial z_{r_{1}}}$ are linearly independent and $\frac{\partial(h \circ V)}{\partial z_{i}}=0$ for $i>r_{1}$.

If $\frac{\partial(g \circ V)}{\partial w_{1}}, \cdots, \frac{\partial(g \circ V)}{\partial w_{r_{1}}}$ are linearly independent, then by Lemma 5.5, we have that $\frac{\partial(k \circ V)}{\partial w_{1}}, \cdots, \frac{\partial(k \circ V)}{\partial w_{r_{1}}}$ are also linearly independent. So $(f \circ V),(g \circ V),(h \circ V)$ and $(k \circ V)$ satisfy conditions (a)-(e) and $f \circ V \overline{g \circ V}-h \circ V \overline{k \circ V}$ satisfies Equation $(5.2)$.

If $\frac{\partial(g \circ V)}{\partial w_{1}}, \cdots, \frac{\partial(g \circ V)}{\partial w_{r_{1}}}$ are linearly dependent, let

$$
L=\left\{a \in C^{r_{1}}: \sum_{i=1}^{r_{1}} a_{i} \frac{\partial(g \circ V)}{\partial w_{i}}=0\right\} .
$$

Using the same argument as in the proof of Lemma 4.2 , we see that there is an $r_{1}$ by $r_{1}$ unitary matrix $U_{2}$ such that $\left\{\frac{\partial\left(g \circ V \circ U_{1}\right)}{\partial w_{1}}, \cdots, \frac{\partial\left(g \circ V \circ U_{1}\right)}{\partial w_{r}}\right\}$ are linearly independent and $\frac{\partial\left(g \circ V \circ U_{1}\right)}{\partial w_{i}}=0$ for $i=r+1$ to $r_{1}$, where $U_{1}=U_{2} \oplus I$, and for some integer $0 \leq r \leq r_{1}$. Also by the chain rule, one easily checks that $\frac{\partial\left(f \circ V \circ U_{1}\right)}{\partial z_{1}}, \cdots, \frac{\partial\left(f \circ V \circ U_{1}\right)}{\partial z_{r}}$ are linearly independent. Then $f \circ V \circ U_{1}$ and $g \circ V \circ U_{1}$ satisfy conditions (a), (b) and (e) as required in the above special case. By Lemma 5.5, $h \circ V \circ U_{1}$ and $k \circ V \circ U_{1}$ also satisfy conditions (c), (d) and (e). Moreover, $f \circ V \circ U_{1} \overline{g \circ V \circ U_{1}}-h \circ V \circ U_{1} \overline{k \circ V \circ U_{1}}$ satisfies Equation (5.2), as desired.

Next we turn to solving Equation (5.2) with conditions (a)-(e). Under conditions (a)-(e), Equation (5.2) becomes

$$
\begin{gathered}
\left(\sum_{i=1}^{n} z_{i} \frac{\partial f}{\partial z_{i}}\right) \overline{\left(\sum_{i=1}^{n} w_{i} \frac{\partial g}{\partial w_{i}}\right)}-\left(\sum_{i=1}^{n} z_{i} \frac{\partial h}{\partial z_{i}} \overline{\left(\sum_{i=1}^{n} w_{i} \frac{\partial k}{\partial w_{i}}\right)}\right. \\
=\sum_{i=1}^{r}\left(\frac{\partial f}{\partial z_{i}} \overline{\frac{\partial g}{\partial w_{i}}}-\frac{\partial h}{\partial z_{i}} \overline{\frac{\partial k}{\partial w_{i}}}\right) .
\end{gathered}
$$

The main idea will be to use the linear independence of the derivatives of $f, g, h$ and $k$ to solve Equation (5.7). Let

$$
\mathcal{H}=\left\{c \in C^{r}: c^{*} \nabla_{r} f \in \operatorname{span}\left\{\frac{\partial h}{\partial z_{1}}, \cdots, \frac{\partial h}{\partial z_{r}}\right\}\right\},
$$

where $\nabla_{r} f=\left(\frac{\partial f}{\partial z_{1}}, \cdots, \frac{\partial f}{\partial z_{r}}\right)^{T}$. We consider three cases.

Case 1 . $\operatorname{dim} \mathcal{H}=r$. In this case there is a matrix $A$ such that

$$
\nabla_{r} f=A \nabla_{r} h \text {. }
$$

Jordan theory in linear algebra implies that there is a unitary matrix $U$ such that

$$
U^{T} A\left(U^{T}\right)^{*}=J_{1} \oplus \cdots \oplus J_{k},
$$

where $J_{i}$ is an $s_{i}$ by $s_{i}$ matrix

$$
\left(\begin{array}{cccccc}
c_{i} & 0 & 0 & \cdots & 0 & 0 \\
1 & c_{i} & 0 & \cdots & 0 & 0 \\
\vdots & \vdots & \vdots & \vdots & \vdots & 0 \\
0 & 0 & 0 & \cdots & 1 & c_{i}
\end{array}\right)
$$


and $\sum_{i=1}^{k} s_{i}=r$. For the sake of simplicity, we still use $f, g, h$, and $k$ to denote respectively $f \circ U, g \circ U, h \circ U$ and $k \circ U$. Using the above Jordan form of $A$ and the chain rule, we have the following equations:

$$
\frac{\partial f}{\partial z_{1}}=c_{1} \frac{\partial h}{\partial z_{1}}, \frac{\partial f}{\partial z_{2}}=c_{1} \frac{\partial h}{\partial z_{2}}+\frac{\partial h}{\partial z_{1}}, \ldots
$$

Taking the derivative of both sides of Equation (5.7) with respect to $z_{1}$, along with the first equation above, we obtain the following equation:

$$
\left(\frac{\partial}{\partial z_{1}} \sum_{i=1}^{n} z_{i} \frac{\partial h}{\partial z_{i}}\right) \overline{\left(\sum_{i=1}^{n} w_{i} \frac{\partial\left(c_{1} g-l\right)}{\partial w_{i}}\right)}=\frac{\partial}{\partial z_{1}} \sum_{i=1}^{n} \frac{\partial h}{\partial z_{i}} \overline{\frac{\partial\left(c_{1} g-k\right)}{\partial w_{i}}} .
$$

By Lemma 4.7, $c_{1} g-k$ is constant, so $\frac{\partial k}{\partial w_{i}}=c_{1} \frac{\partial g}{\partial w_{i}}$. Also $c_{1}$ is zero because $k$ is not constant. Replacing $\frac{\partial k}{\partial w_{i}}$ by $c_{1} \frac{\partial g}{\partial w_{i}}$ in Equation (5.7), we have

$$
\frac{\partial}{\partial z_{1}}\left(\sum_{i=1}^{n} z_{i} \frac{\partial\left(f-\overline{c_{1}} h\right)}{\partial z_{i}}\right) \overline{\left(\sum_{i=1}^{n} w_{i} \frac{\partial g}{\partial w_{i}}\right)}=\frac{\partial}{\partial z_{1}} \sum_{i=1}^{n} \frac{\partial\left(f-\overline{c_{1}} h\right)}{\partial z_{i}} \frac{\overline{\partial g}}{\partial w_{i}}
$$

By Lemma 4.7 again, $f-\overline{c_{1}} h$ is constant. So $f, g, h$, and $k$ satisfy condition (5).

Case 2. $\operatorname{dim} \mathcal{H}<r-1$. In this case, using the same argument as in the proof of Lemma 4.2, we may assume that $\left\{\frac{\partial f}{\partial z_{1}}, \cdots, \frac{\partial f}{\partial z_{j}}\right\}$ are linearly independent of $\left\{\frac{\partial h}{\partial z_{1}}, \cdots, \frac{\partial h}{\partial z_{r}}\right\}$ and $\left\{\frac{\partial f}{\partial z_{j+1}}, \cdots, \frac{\partial f}{\partial z_{r}}\right\}$ are in the $\operatorname{span}\left\{\frac{\partial h}{\partial z_{1}}, \cdots, \frac{\partial h}{\partial z_{r}}\right\}$ for some $j \geq 2$. Then by Equation (5.7), there are vectors $a, a^{\prime}, c, c^{\prime}$ and a matrix $B$ such that

$$
\begin{gathered}
\sum_{i=1}^{n} z_{i} \frac{\partial f}{\partial z_{i}}=a^{*} \nabla_{j} f+c^{*} \nabla_{r} h, \\
\sum_{i=1}^{n} z_{i} \frac{\partial h}{\partial z_{i}}=\left(a^{\prime}\right)^{*} \nabla_{j} f+\left(c^{\prime}\right)^{*} \nabla_{r} h,
\end{gathered}
$$

and

$$
\left(\frac{\partial f}{\partial z_{j+1}}, \cdots, \frac{\partial f}{\partial z_{n}}\right)^{T}=\left(\nabla_{r} h\right)^{T} B^{*} .
$$

Combining Equation (5.7) with the above equations, and using the fact that $\frac{\partial f}{\partial z_{1}}$, $\cdots, \frac{\partial f}{\partial z_{j}}, \frac{\partial h}{\partial z_{1}}, \cdots, \frac{\partial h}{\partial z_{r}}$ are linearly independent, we obtain

$$
a \sum_{i=1}^{n} w_{i} \frac{\partial g}{\partial w_{i}}-a^{\prime} \sum_{i=1}^{n} w_{i} \frac{\partial k}{\partial w_{i}}=\nabla_{j} g
$$

and

$$
c \sum_{i=1}^{n} w_{i} \frac{\partial g}{\partial w_{i}}-c^{\prime} \sum_{i=1}^{n} w_{i} \frac{\partial k}{\partial w_{i}}=B\left(\frac{\partial g}{\partial w_{j+1}}, \cdots, \frac{\partial g}{\partial w_{n}}\right)^{T}-\nabla_{r} k .
$$

Since $\frac{\partial g}{\partial w_{1}}, \cdots, \frac{\partial g}{\partial w_{j}}$ are linearly independent, the first system of equations above gives that $\sum_{i=1}^{n} w_{i} \frac{\partial g}{\partial w_{i}}$ and $\sum_{i=1}^{n} w_{i} \frac{\partial k}{\partial w_{i}}$ are in the $\operatorname{span}\left\{\frac{\partial g}{\partial w_{1}}, \cdots, \frac{\partial g}{\partial w_{r}}\right\}$. The second system of equations above gives that $\left\{\frac{\partial k}{\partial w_{1}}, \cdots, \frac{\partial k}{\partial w_{r}}\right\}$ are in the $\operatorname{span}\left\{\frac{\partial g}{\partial w_{1}}, \cdots, \frac{\partial g}{\partial w_{r}}\right\}$. So this case is reduced to Case 1 if we replace $f$ and $h$ by $g$ and $k$ respectively. 
Case 3. $\operatorname{dim} \mathcal{H}=r-1$. Using the same argument as in the proof of Lemma 4.2 , we may assume that $\frac{\partial f}{\partial z_{1}}, \frac{\partial h}{\partial z_{1}}, \cdots, \frac{\partial h}{\partial z_{r}}$ are linearly independent, and there is an $(r-1) \times r$ matrix $A$ such that

$$
\left(\frac{\partial f}{\partial z_{2}}, \cdots, \frac{\partial f}{\partial z_{r}}\right)^{T}=A \nabla_{r} h .
$$

If there is a constant $b$ such that $b g-k$ is constant, then $\frac{\partial k}{\partial w_{i}}=b \frac{\partial g}{\partial w_{i}}$. For each $1 \leq j \leq n$, differentiating (5.7) with respect to $z_{j}$ gives

$$
\frac{\partial}{\partial z_{j}}\left(\sum_{i=1}^{n} z_{i} \frac{\partial(f-b h)}{\partial z_{i}}\right) \overline{\left(\sum_{i=1}^{n} w_{i} \frac{\partial g}{\partial w_{i}}\right)}=\frac{\partial}{\partial z_{j}} \sum_{i=1}^{n} \frac{\partial(f-b h)}{\partial z_{i}} \frac{\overline{\partial g}}{\partial w_{i}} .
$$

By Lemma $4.7, \frac{\partial(f-b h)}{\partial z_{j}}=0$ for $j$ and so $f-b h$ is constant. Thus $f, g, h$ and $k$ satisfy condition (5).

Suppose that there is no constant $b$ such that $b g-k$ is constant. We will derive a contradiction. By Equation (5.7), there are constants $c_{1}, c_{2}$ and vectors $a, a^{\prime}$ such that

$$
\sum_{i=1}^{n} z_{i} \frac{\partial f}{\partial z_{i}}=c_{1} \frac{\partial f}{\partial z_{1}}+a^{*} \nabla_{r} h
$$

and

$$
\sum_{i=1}^{n} z_{i} \frac{\partial h}{\partial z_{i}}=c_{2} \frac{\partial f}{\partial z_{1}}+\left(a^{\prime}\right)^{*} \nabla_{r} h
$$

Extend the holomorphic function $f$ on $B_{n}$ to a holomorphic function $\tilde{f}$ on $B_{n+1}$ by

$$
\tilde{f}\left(z_{1}, \cdots, z_{n}, z_{n+1}\right)=f\left(z_{1}, \cdots, z_{n}\right) .
$$

By Lemma 5.1, $\tilde{f} \overline{\tilde{g}}-\tilde{h} \overline{\tilde{k}}$ is $\mathcal{M}$-harmonic on $B_{n+1}$.

For $c$ in the unit disk, define

$$
\varphi_{c}\left(z_{1}, \cdots, z_{n}, z_{n+1}\right)=\left(\frac{z_{1} s}{1-\bar{c} z_{n+1}}, \cdots, \frac{z_{n} s}{1-\bar{c} z_{n+1}}, \frac{c-z_{n+1}}{1-\bar{c} z_{n+1}}\right),
$$

where $s=\left(1-|c|^{2}\right)^{1 / 2}$; then $\varphi_{c} \in \operatorname{Aut}\left(B_{n+1}\right)$. Note that the space of $\mathcal{M}$-harmonic functions is invariant under the action of $\operatorname{Aut}\left(B_{n+1}\right)$. Then

$$
\tilde{f} \circ \varphi_{c} \overline{\tilde{g} \circ \varphi_{c}}-\tilde{h} \circ \varphi_{c} \overline{\tilde{l} \circ \varphi_{c}}
$$

is $\mathcal{M}$-harmonic on $B_{n+1}$.

By the chain rule, we have

$$
\frac{\partial\left(\tilde{f} \circ \varphi_{c}\right)}{\partial z_{n+1}}=\sum_{i=1}^{n} \frac{\bar{c} s z_{i}}{\left(1-\bar{c} z_{n+1}\right)^{2}} \frac{\partial \tilde{f}}{\partial z_{i}} \circ \varphi_{c}
$$

and

$$
\frac{\partial\left(\tilde{f} \circ \varphi_{c}\right)}{\partial z_{j}}=\frac{s}{\left(1-\bar{c} z_{n+1}\right)} \frac{\partial \tilde{f}}{\partial z_{j}} \circ \varphi_{c}
$$

for $j<n+1$. Thus (5.11) gives

$$
\frac{\partial\left(\tilde{f} \circ \varphi_{c}\right)}{\partial z_{n+1}}=\frac{c}{\left(1-\bar{c} z_{n+1}\right)} \sum_{i=1}^{n}\left(\varphi_{c}\right)_{i} \frac{\partial f}{\partial z_{i}}\left(\left(\varphi_{c}(z)\right)_{i}, \cdots,\left(\varphi_{c}(z)\right)_{n}\right)
$$


Since $\sum_{i=1}^{n} z_{i} \frac{\partial f}{\partial z_{i}}=c_{1} \frac{\partial f}{\partial z_{1}}+a^{*} \nabla_{r} h$, we have

$$
\begin{aligned}
& \sum_{i=1}^{n}\left(\varphi_{c}\right)_{i} \frac{\partial f}{\partial z_{i}}\left(\left(\varphi_{c}(z)\right)_{i}, \cdots,\left(\varphi_{c}(z)\right)_{n}\right) \\
& \quad=c_{1} \frac{\partial \tilde{f}}{\partial z_{1}} \circ \varphi_{c}+a^{*} \nabla_{r} h\left(\left(\varphi_{c}(z)\right)_{i}, \cdots,\left(\varphi_{c}(z)\right)_{n}\right) .
\end{aligned}
$$

Then (5.13) combined with (5.12) gives

$$
\frac{\partial\left(\tilde{f} \circ \varphi_{c}\right)}{\partial z_{n+1}}=\frac{\bar{c}}{s} c_{1} \frac{\partial\left(\tilde{f} \circ \varphi_{c}\right)}{\partial z_{1}}+\frac{\bar{c}}{s} a^{*} \nabla_{r}\left(\tilde{h} \circ \varphi_{c}\right) .
$$

Similarly we can show that

$$
\frac{\partial\left(\tilde{h} \circ \varphi_{c}\right)}{\partial z_{n+1}}=\frac{\bar{c}}{s} c_{2} \frac{\partial\left(\tilde{f} \circ \varphi_{c}\right)}{\partial z_{1}}+\frac{\bar{c}}{s}\left(a^{\prime}\right)^{*} \nabla_{r}\left(\tilde{h} \circ \varphi_{c}\right) .
$$

(i) If $c_{2} \neq 0$, let $F=\tilde{f} \circ \varphi_{c}, G=\tilde{g} \circ \varphi_{c}, H=\tilde{h} \circ \varphi_{c}$ and $K=\tilde{k} \circ \varphi_{c}$; then (5.14) and (5.15) give that $\frac{\partial F}{\partial z_{i}}$ is in the $\operatorname{span}\left\{\frac{\partial H}{\partial z_{1}}, \cdots, \frac{\partial H}{\partial z_{r}}, \frac{\partial H}{\partial z_{n+1}}\right\}$ for $i=1, \cdots, r$, and $n+1$. Since $F \bar{G}-H \bar{K}$ is $\mathcal{M}$-harmonic on $B_{n+1}$, by Lemma 5.1 , we have

$$
\begin{aligned}
& {\left[\sum_{i=1}^{n+1} z_{i} \frac{\partial F}{\partial z_{i}}\right]\left[\overline{\sum_{i=1}^{n+1} w_{i} \frac{\partial G}{\partial w_{i}}}\right]-\left[\sum_{i=1}^{n+1} z_{i} \frac{\partial H}{\partial z_{i}}\right]\left[\overline{\sum_{i=1}^{n+1} w_{i} \frac{\partial K}{\partial w_{i}}}\right]} \\
& =\sum_{i=1}^{r}\left(\frac{\partial F}{\partial z_{i}} \overline{\frac{\partial G}{\partial w_{i}}}-\frac{\partial H}{\partial z_{i}} \overline{\frac{\partial K}{\partial w_{i}}}\right)-\left(\frac{\partial F}{\partial z_{n+1}} \overline{\frac{\partial G}{\partial w_{n+1}}}-\frac{\partial H}{\partial z_{n+1}} \overline{\frac{\partial K}{\partial w_{n+1}}}\right) .
\end{aligned}
$$

because

$$
\sum_{i=r+1}^{n}\left(\frac{\partial F}{\partial z_{i}} \overline{\frac{\partial G}{\partial w_{i}}}-\frac{\partial H}{\partial z_{i}} \overline{\frac{\partial K}{\partial w_{i}}}\right)=0
$$

Thus this case is reduced to Case 1 . Hence $F, G, H$ and $K$ satisfy condition (5) of this theorem. So do $f, g, h$, and $k$. But this contradicts that there is no constant $b$ such that $b g-k$ is constant.

(ii) If $c_{2}=0$, then (5.10) becomes

$$
\sum_{i=1}^{n} z_{i} \frac{\partial h}{\partial z_{i}}=\left(a^{\prime}\right)^{*} \nabla_{r} h
$$

Replacing $\sum_{i=1}^{n} z_{i} \frac{\partial h}{\partial z_{i}}$ in Equation (5.7) by the right hand side of the above equation gives

$$
\overline{c_{1}} \sum_{i=1}^{n} w_{i} \frac{\partial g}{\partial w_{i}}=\frac{\partial g}{\partial w_{1}}
$$

and

$$
a \sum_{i=1}^{n} w_{i} \frac{\partial g}{\partial w_{i}}-a^{\prime} \sum_{i=1}^{n} w_{i} \frac{\partial k}{\partial w_{i}}=A^{*}\left(\frac{\partial g}{\partial w_{2}}, \cdots, \frac{\partial g}{\partial w_{r}}\right)^{T}-\nabla_{r} k
$$

These equations come from linear independence of $\frac{\partial f}{\partial z_{1}}, \frac{\partial h}{\partial z_{1}}, \cdots, \frac{\partial h}{\partial z_{r}}$.

By the chain rule, (5.16) gives

$$
\overline{c_{1}} \frac{\partial\left(\tilde{g} \circ \varphi_{c}\right)}{\partial w_{n+1}}=\frac{\bar{c}}{s} \frac{\partial\left(\tilde{g} \circ \varphi_{c}\right)}{\partial w_{1}},
$$


which means

$$
\overline{c_{1}} \frac{\partial G}{\partial w_{n+1}}=\frac{\bar{c}}{s} \frac{\partial G}{\partial w_{1}}
$$

Since neither $F$ nor $H$ is constant, Lemma 5.5 implies

$$
\overline{c_{1}} \frac{\partial K}{\partial w_{n+1}}=\frac{\bar{c}}{s} \frac{\partial K}{\partial w_{1}}
$$

and so

$$
\overline{c_{1}} \frac{\partial\left(\tilde{k} \circ \varphi_{c}\right)}{\partial w_{n+1}}=\frac{\bar{c}}{s} \frac{\partial\left(\tilde{k} \circ \varphi_{c}\right)}{\partial w_{1}} .
$$

Then by the chain rule again, we obtain

$$
\overline{c_{1}} \sum_{i=1}^{n} w_{i} \frac{\partial k}{\partial w_{i}}=\frac{\partial k}{\partial w_{1}} .
$$

Putting the above equation, (5.15) and (5.17) together gives

$$
\left(\frac{a}{\overline{c_{1}}},-A^{*}\right) \nabla_{r} g=\left(\begin{array}{ccccc}
\frac{a_{1}^{\prime}}{\overline{c_{1}}}-1 & 0 & 0 & \cdots & 0 \\
\frac{a_{2}^{\prime}}{\overline{c_{1}}} & -1 & 0 & \cdots & 0 \\
\vdots & \vdots & \vdots & \vdots & \vdots \\
\frac{a_{r}^{\prime}}{\overline{c_{1}}} & o & 0 & \cdots & -1
\end{array}\right) \nabla_{r} k .
$$

If $\frac{a_{1}^{\prime}}{c_{1}}-1 \neq 0$, the system of equations above gives that $\frac{\partial k}{\partial w_{i}}$ is in the space spanned by $\left\{\frac{\partial g}{\partial w_{1}}, \cdots, \frac{\partial g}{\partial w_{r}}\right\}$, so this case is reduced to Case 1 for $g$ and $k$. This contradicts that there is no constant $b$ such that $b g-k$ is constant.

If $\frac{a_{1}^{\prime}}{c_{1}}-1=0$, the system of equations above gives

$$
\frac{a_{1}}{\overline{c_{1}}} \frac{\partial g}{\partial w_{1}}-\overline{a_{21}} \frac{\partial g}{\partial w_{2}}-\cdots-\overline{a_{r 1}} \frac{\partial g}{\partial w_{r}}=0
$$

Note that $\left\{\frac{\partial g}{\partial w_{1}}, \cdots, \frac{\partial g}{\partial w_{r}}\right\}$ are linearly independent. Then $a_{i 1}=0$ for $i=2, \cdots, r$. By (5.8), we have

$$
\left(\frac{\partial f}{\partial z_{2}}, \cdots, \frac{\partial f}{\partial z_{r}}\right)^{T}=\left(0, A_{(r-1) \times(r-1)}\right) \nabla_{r} h .
$$

So

$$
\left(\frac{\partial f}{\partial z_{2}}, \cdots, \frac{\partial f}{\partial z_{r}}\right)^{T}=A_{(r-1) \times(r-1)}\left(\frac{\partial h}{\partial z_{2}}, \cdots, \frac{\partial h}{\partial z_{r}}\right)^{T} .
$$

Without loss of generality, we may assume that

$$
A=J_{1} \oplus \cdots \oplus J_{k_{1}}
$$

where $J_{i}$ is the $s_{i}$ by $s_{i}$ matrix

$$
\left(\begin{array}{cccccc}
c_{i} & 0 & 0 & \cdots & 0 & 0 \\
1 & c_{i} & 0 & \cdots & 0 & 0 \\
\vdots & \vdots & \vdots & \vdots & \vdots & 0 \\
0 & 0 & 0 & \cdots & 1 & c_{i}
\end{array}\right)
$$


and $\sum_{i=1}^{k_{1}} s_{i}=r-1$. Using the Jordan form of $A$, we get

$$
\frac{\partial f}{\partial z_{2}}=c_{1} \frac{\partial h}{\partial z_{2}}
$$

Taking the derivative of both sides of Equation (5.7) with respect to $z_{2}$, and using the above equation, we have

$$
\frac{\partial}{\partial z_{2}}\left(\sum_{i=1}^{n} z_{i} \frac{\partial h}{\partial z_{i}}\right) \overline{\left(\sum_{i=1}^{r} w_{i} \frac{\partial\left(\overline{c_{1}} g-k\right)}{\partial w_{i}}\right)}=\frac{\partial}{\partial z_{2}} \sum_{i=1}^{n} \frac{\partial h}{\partial z_{i}} \frac{\overline{\partial\left(\overline{c_{1}} g-k\right)}}{\partial w_{i}} .
$$

By Lemma 4.7, $\overline{c_{1}} g-k$ is constant. This contradicts that there is no constant $b$ such that $b g-k$ is constant. This completes the proof.

To end this section, we restate Theorem 5.6 as follows.

Corollary 5.18. Suppose that $f, g, h$ and $k$ are in $H^{2 n}\left(B_{n}\right)$. Then $f \bar{g}-h \bar{k}$ is $\mathcal{M}$-harmonic if and only if it is pluriharmonic.

\section{Proofs of main Results}

In this section we will present the proof of the main results. We begin with a simple fact about bounded pluriharmonic functions on $B_{n}$.

Proposition 6.1. If $f$ is a bounded pluriharmonic function on $B_{n}$, then there are functions $f_{1}$ and $f_{2}$ in $H^{p}\left(S_{n}\right)$ for all $p>1$ such that $f=f_{1}+\bar{f}_{2}$.

Proof. Without loss of generality, we consider just the case when $f$ is real-valued. Because $f$ is pluriharmonic, there is a holomorphic function $f_{1}$ on $B_{n}$ such that $f=f_{1}+\bar{f}_{1}$. We need to show that $f_{1}$ is in $H^{p}\left(S_{n}\right)$ for all $p>1$. It is easy to check that $S(f)=f_{1}+\bar{f}_{1}(0)$, where $S$ is the Hardy projection. It is well-known that the Hardy projection $S$ is bounded on $L^{p}\left(S_{n}\right)$ for any $p>1$. Note that $f$ is in $L^{\infty}\left(B_{n}\right)$. Then $f_{1}$ is in $H^{p}\left(S_{n}\right)$ for any $p>1$. This completes the proof.

The following theorem easily follows from recent result of Ahern, Flores and Rudin [AFR] for $n<12$. The result here is valid for all $n$. It was also obtained by a different method in [CL]. Our method extends the method in $[\mathrm{AxC}]$ and [Zh1] directly.

Theorem 6.2. Suppose that $f, g, h$ and $k$ are in $H^{2}\left(S_{n}\right)$ with the property

$$
\int_{B_{n}}(f \bar{g}-h \bar{k}) \circ \psi(w)\left|k_{z}(w)\right|^{2} d A(w)=(f \bar{g}-h \bar{k}) \circ \psi(z)
$$

for all $\psi \in \operatorname{Aut}\left(B_{n}\right)$. Then $(f \bar{g}-h \bar{k})$ is $\mathcal{M}$-harmonic on the unit ball $B_{n}$.

Proof. Let $d \mathcal{U}$ be the Haar measure on the group $\mathcal{U}$ of $n \times n$ unitary matrices. Define

$$
G(z)=\int_{\mathcal{U}}(f \bar{g}-h \bar{k})(U z) d \mathcal{U}
$$

for $z$ in $B_{n}$. For any $f$ in $H^{2}(S)$, write $f$ as a power series

$$
f(z)=\sum_{\alpha} a_{\alpha}(f) z^{\alpha}
$$

with

$$
\sum_{\alpha}\left|a_{\alpha}(f)\right|^{2} I(\alpha)<+\infty
$$

where $I(\alpha)=\left\|z^{\alpha}\right\|_{H^{2}(S)}^{2}$. 
As $\mathcal{U}$ is a subgroup of $\operatorname{Aut}\left(B_{n}\right)$, for any $U \in \mathcal{U}$, letting $\psi=U$ in (6.3), we have

$$
\int_{B_{n}}(f \bar{g}-h \bar{k}) \circ U(w)\left|k_{z}(w)\right|^{2} d A(w)=(f \bar{g}-h \bar{k}) \circ U(z) .
$$

Integrating both sides of the above equation with respect to $U$ over $\mathcal{U}$ gives

$$
\int_{B} G(w)\left|k_{z}(w)\right|^{2} d A(w)=G(z) .
$$

By the formula (3.2), we have

$$
\begin{gathered}
G(z)=\int_{S}(f \bar{g}-h \bar{k})(\|z\| \xi) d \sigma(\xi) \\
=\sum_{\alpha}\left(a_{\alpha}(f) \overline{a_{\alpha}(g)}-a_{\alpha}(h) \overline{a_{\alpha}(k)}\right) I(\alpha)\left\|z^{\alpha}\right\|^{2|\alpha|},
\end{gathered}
$$

where $|\alpha|=\sum_{i=1}^{n} \alpha_{i}$.

Since $f, g, h$ and $k$ are in $H^{2}\left(S_{n}\right), G(z)$ is continuous on $\bar{B}_{n}$. The mean value theorem tells us that $\tilde{\Delta} G=0$. In addition, $G(z)$ is constant on $S_{n}$. It follows from the maximal principle that $G(z)$ is constant on $B_{n}$. Thus

$$
\sum_{|\alpha|=1}\left[a_{\alpha}(f) \overline{a_{\alpha}(g)}-a_{\alpha}(h) \overline{a_{\alpha}(k)}\right]=0 .
$$

For convenience we let $a_{i}(f)$ denote $a_{(0, \cdots, 0,1,0, \cdots, 0)}(f)$. Then Equation $(6.5)$ becomes

$$
\sum_{i}\left[a_{i}(f) \overline{a_{i}(g)}-a_{i}(h) \overline{a_{i}(k)}\right]=0 .
$$

By the chain rule, $a_{i}(f)=\frac{\partial f}{\partial z_{i}}(0)$. Letting $a_{i}(f)(w)=\frac{\partial\left(f \circ \varphi_{w}\right)}{\partial z_{i}}(0)$, similarly we get that

$$
\sum_{i}\left[a_{i}(f)(z) \overline{a_{i}(g)(z)}-a_{i}(h)(z) \overline{a_{i}(z)}\right]=0 .
$$

The above equation comes from the fact that the space of $\mathcal{M}$-harmonic functions is invariant under the action of $A u t\left(B_{n}\right)$.

Calculating $a_{i}(f)(w)$ by the chain rule, we obtain

$$
a(f)(z)=s(1-s) P_{\bar{z}} \nabla_{z} f-s \nabla_{z} f,
$$

where $s=\left(1-\|z\|^{2}\right)^{1 / 2}$ and

$$
a(f)(z)=\left(\begin{array}{c}
a_{1}(f)(z) \\
\vdots \\
a_{n}(f)(z)
\end{array}\right) .
$$

Replacing $a_{i}(f)(z)$ in (6.6) by the right hand side of (6.7) gives

$$
\begin{gathered}
0=\left[s(1-s) P_{\bar{z}} \nabla_{z} g-s \nabla_{z} g\right]^{*}\left[s(1-s) P_{\bar{z}} \nabla_{z} f-s \nabla_{z} f\right] \\
-\left[s(1-s) P_{\bar{z}} \nabla_{z} k-s \nabla_{z} k\right]^{*}\left[s(1-s) P_{\bar{z}} \nabla_{z} h-s \nabla_{z} h\right] .
\end{gathered}
$$


Simplifying the above equation yields

$$
\begin{aligned}
& -s^{2}\left(1-s^{2}\right)\langle\bar{z}, \nabla g\rangle\langle\nabla f, \bar{z}\rangle+s^{2}\left(1-s^{2}\right)\langle\nabla f, \nabla g\rangle \\
& \quad=-s^{2}\left(1-s^{2}\right)\langle\bar{z}, \nabla k\rangle\langle\nabla h, \bar{z}\rangle+s^{2}\left(1-s^{2}\right)\langle\nabla h, \nabla k\rangle .
\end{aligned}
$$

Multiplying both sides of the above equation by $\frac{1}{s^{2}\left(1-s^{2}\right)}$, we obtain

$$
\begin{gathered}
\langle\bar{z}, \nabla g\rangle\langle\nabla f, \bar{z}\rangle-\langle\bar{z}, \nabla k\rangle\langle\nabla h, \bar{z}\rangle \\
=\langle\nabla f, \nabla g\rangle-\langle\nabla h, \nabla k\rangle .
\end{gathered}
$$

We conclude that

$$
\begin{gathered}
\left(\sum_{j=1}^{n} z_{j} \frac{\partial f}{\partial z_{j}}\right)\left(\sum_{i=1}^{n} \overline{z_{i} \frac{\partial g}{\partial z_{i}}}\right)-\left(\sum_{j=1}^{n} z_{j} \frac{\partial h}{\partial z_{j}}\right)\left(\sum_{i} \overline{z_{i} \frac{\partial k}{\partial z_{i}}}\right) \\
=\sum_{j=1}^{n} \frac{\partial f}{\partial z_{j}} \frac{\overline{\partial g}}{\partial z_{j}}-\sum_{j=1}^{n} \frac{\partial h}{\partial z_{j}} \overline{\frac{\partial k}{\partial z_{j}}}
\end{gathered}
$$

which is Equation (3.1). So $f \bar{g}-h \bar{k}$ is $\mathcal{M}$-harmonic. This completes the proof.

Lemma 6.8. Let $f, g, h$, and $k$ be holomorphic on the unit ball $B_{n}$. Suppose that $\varphi=f+\bar{k}$ and $\psi=h+\bar{g}$. Then $\varphi$ and $\psi$ satisfy one of conditions (1)-(4) in Theorem 2.1 if and only if $f, g, h$, and $k$ satisfy one of conditions (1)-(5) in Theorem 5.6.

The proof of Lemma 6.8 is easy. We leave it as an exercise to readers.

For each $z \in B_{n}$, define an operator $U_{z}$ on the Bergman space $L_{a}^{2}\left(B_{n}\right)$ by

$$
U_{z} u=u \circ \varphi_{z} k_{z}
$$

where $\varphi_{z}$ is the Möbius transformation and $k_{z}$ is the normalized reproducing kernel. If $V$ is unitary matrix in $C^{n}$, define an operator $U_{V}$ on $L_{a}^{2}\left(B_{n}\right)$ by $U_{V} u=u \circ V$. A simple computation shows that $U_{z}$ and $U_{V}$ are a unitary operators from $L_{a}^{2}\left(B_{n}\right)$ onto $L_{a}^{2}\left(B_{n}\right)$. So for each element $\tau$ in $\operatorname{Aut}\left(B_{n}\right)$, we can associate a unitary operator $U_{\tau}$ on $L_{a}^{2}\left(B_{n}\right)$ with $\tau$ by $U_{\tau}=U_{V} U_{z}$ if $\tau=U \circ \varphi_{z}$. Immediately we see that

$$
U_{\tau} T_{f} U_{\tau}^{*}=T_{f \circ \tau} \text {. }
$$

Hence

$$
U_{\tau}\left(T_{\varphi} T_{\psi}-T_{\psi} T_{\varphi}\right) U_{\tau}^{*}=\left(T_{\varphi \circ \tau} T_{\psi \circ \tau}-T_{\psi \circ \tau} T_{\varphi \circ \tau}\right) .
$$

We need the following lemma in the proof of Theorem 2.1.

Lemma 6.10. Let $f, g, h$, and $k$ be holomorphic on the unit ball $B_{n}$. Suppose that $\varphi=f+\bar{k}$ and $\psi=h+\bar{g}$ are bounded on $B_{n}$. Then

$$
\begin{gathered}
\left\langle\left(T_{\varphi \circ \tau} T_{\psi \circ \tau}-T_{\psi \circ \tau} T_{\varphi \circ \tau}\right) k_{z}, k_{z}\right\rangle \\
=-\int_{B_{n}}(f \circ \tau(w) \overline{g \circ \tau(w)}-h \circ \tau(w) \overline{k \circ \tau(w)})\left|k_{z}(w)\right|^{2} d A(w) \\
+(f \circ \tau(z) \overline{g \circ \tau(z)}-h \circ \tau(z) \overline{k \circ \tau(z)})
\end{gathered}
$$

for each $\tau \in \operatorname{Aut}\left(B_{n}\right)$. 
Proof. Suppose that

$$
\varphi=f+\bar{k} \text { and } \psi=h+\bar{g} .
$$

Since

$$
T_{\varphi} 1=P(\varphi)=P(f+\bar{k})=f+\overline{k(0)},
$$

we have

$$
\begin{gathered}
\left\langle T_{\psi} T_{\varphi} 1,1\right\rangle=\left\langle T_{\varphi} 1, T_{\bar{\psi}} 1\right\rangle=\left\langle T_{\varphi} 1, \bar{\psi}\right\rangle=\langle(f+\overline{k(0)}), \bar{\psi}\rangle=\langle(\varphi+\overline{k(0)}-\bar{k}), \bar{\psi}\rangle \\
=\langle\varphi, \bar{\psi}\rangle+\langle(\overline{k(0)}-\bar{k}), \bar{\psi}\rangle=\langle\psi \varphi, 1\rangle+\overline{k(0)} h(0)-\langle\bar{k} h, 1\rangle .
\end{gathered}
$$

Interchanging $\varphi$ and $\psi$ in the above equation gives

$$
\left\langle T_{\varphi} T_{\psi} 1,1\right\rangle=\langle\varphi \psi, 1\rangle+\overline{g(0)} f(0)-\langle\bar{g} f, 1\rangle .
$$

Hence we obtain

$$
\left\langle\left(T_{\varphi} T_{\psi}-T_{\psi} T_{\varphi}\right) 1,1\right\rangle=\overline{g(0)} f(0)-\overline{k(0)} h(0)-[\langle\bar{g} f, 1\rangle-\langle\bar{k} h, 1\rangle] .
$$

For each $\tau \in \operatorname{Aut}\left(B_{n}\right)$, composing both sides of Equation (6.12) with $\tau$ expresses each of the functions $\varphi \circ \tau$ and $\psi \circ \tau$ as the sum of a holomorphic function and the conjugate of a holomorphic function:

$$
\varphi \circ \tau=f \circ \tau+\overline{k \circ \tau} \text { and } \psi \circ \tau=h \circ \tau+\overline{g \circ \tau} .
$$

Note that $\tau \circ \varphi_{z}$ is still in $\operatorname{Aut}\left(B_{n}\right)$ for each $\tau \in A u t\left(B_{n}\right)$ and $z \in B_{n}$. Replacing each function in (6.13) by its composition with $\tau \circ \varphi_{z}$ gives

$$
\begin{gathered}
\left\langle\left(T_{\varphi \circ \tau \circ \varphi_{z}} T_{\psi \circ \tau \circ \varphi_{z}}-T_{\psi \circ \tau \circ \varphi_{z}} T_{\varphi \circ \tau \circ \varphi_{z}}\right) 1,1\right\rangle \\
=-\int_{B_{n}}\left(f \circ \tau \circ \varphi_{z}(w) \overline{g \circ \tau \circ \varphi_{z}(w)}-h \circ \tau \circ \varphi_{z}(w) \overline{k \circ \tau \circ \varphi_{z}(w)}\right) d A(w) \\
\quad+\left(f \circ \tau \circ \varphi_{z}(0) \overline{g \circ \tau \circ \varphi_{z}(0)}-h \circ \tau \circ \varphi_{z}(0) \overline{k \circ \tau \circ \varphi_{z}(0)}\right) .
\end{gathered}
$$

On the other hand, $U_{z} 1=k_{z}$. By (6.9), we have

$$
\left\langle\left(T_{\varphi \circ \tau \circ \varphi_{z}} T_{\psi \circ \tau \circ \varphi_{z}}-T_{\psi \circ \tau \circ \varphi_{z}} T_{\varphi \circ \tau \circ \varphi_{z}}\right) 1,1\right\rangle=\left\langle\left(T_{\varphi \circ \tau} T_{\psi \circ \tau}-T_{\psi \circ \tau} T_{\varphi \circ \tau}\right) k_{z}, k_{z}\right\rangle .
$$

Making the change of variables $\lambda=\varphi_{z}(w)$, we get

$$
\begin{gathered}
\int_{B_{n}}\left(f \circ \tau \circ \varphi_{z}(w) \overline{g \circ \tau \circ \varphi_{z}(w)}-h \circ \tau \circ \varphi_{z}(w) \overline{k \circ \tau \circ \varphi_{z}(w)}\right) d A(w) \\
=\int_{B_{n}}(f \circ \tau(\lambda) \overline{g \circ \tau(\lambda)}-h \circ \tau(\lambda) \overline{k \circ \tau(\lambda)})\left|k_{z}(\lambda)\right|^{2} d A(\lambda) .
\end{gathered}
$$

Combining (6.14) with (6.15) and (6.16), we obtain (6.11), as desired. This completes the proof.

Now we are ready to prove Theorems 2.1 and 2.2 . 
Proof of Theorem 2.1. First we prove that if $\varphi$ and $\psi$ satisfy one of the conditions in Theorem 2.1, then $T_{\varphi}$ commutes with $T_{\psi}$.

If $\varphi$ is holomorphic on $B_{n}$, then $T_{\varphi}$ is the operator on the Bergman space $L_{a}^{2}\left(B_{n}\right)$ of multiplication by $\varphi$. So if $\varphi$ and $\psi$ satisfy condition (1), then $T_{\varphi}$ commutes with $T_{\psi}$. Since the adjoint of $T_{\varphi}$ on the Bergman space is $T_{\bar{\varphi}}, T_{\varphi}$ commutes with $T_{\psi}$ if $\varphi$ and $\psi$ satisfy condition (2). If $\varphi$ and $\psi$ satisfy condition (3), either $T_{\varphi}$ or $T_{\psi}$ is a scalar operator, so $T_{\varphi}$ commutes with $T_{\psi}$. If $\varphi$ and $\psi$ satisfy condition (4), let $c=b \psi-\varphi$ be a constant. Thus $T_{\varphi}=b T_{\psi}+c$. So $T_{\varphi}$ commutes with $T_{\psi}$.

To prove the other direction of Theorem 2.1, suppose that $T_{\varphi}$ commutes with $T_{\psi}$ on $L_{a}^{2}\left(B_{n}\right)$. We need to show that $\varphi$ and $\psi$ satisfy one of the conditions in Theorem 2.1. By Proposition 6.1, there are four functions $f, g, h$, and $k$ in $H^{2 n}\left(S_{n}\right)$ such that

$$
\varphi=f+\bar{k} \text { and } \psi=h+\bar{g} .
$$

By Lemma 6.8 , it suffices to show that $f, g, h$, and $k$ satisfy one of the conditions in Theorem 5.6. To do this, we need to show that these functions satisfy Equation (6.3) in Theorem 6.2.

Since $T_{\varphi}$ commutes with $T_{\psi}, T_{\varphi} T_{\psi}-T_{\psi} T_{\varphi}=0$. Then by (6.9), we have

$$
T_{\varphi \circ \tau} T_{\psi \circ \tau}-T_{\psi \circ \tau} T_{\varphi \circ \tau}=0
$$

for each $\tau \in \operatorname{Aut}\left(B_{n}\right)$. Thus it follows from Lemma 6.10 that

$$
\begin{gathered}
\int_{B_{n}}(f \circ \tau(w) \overline{g \circ \tau(w)}-h \circ \tau(w) \overline{k \circ \tau(w)})\left|k_{z}(w)\right|^{2} d A(w) \\
=f \circ \tau(z) \overline{g \circ \tau(z)}-h \circ \tau(z) \overline{k \circ \tau(z)}
\end{gathered}
$$

for each $\tau \in \operatorname{Aut}\left(B_{n}\right)$. So $f, g, h$ and $k$ satisfy the condition of Theorem 6.2 , as desired. This completes the proof.

Proof of Theorem 2.2. The proof of the "if" part is similar to that in the proof of Theorem 2.1, so it is omitted. It remains to prove the "only if" part.

Suppose that $T_{\varphi}$ commutes with $T_{\psi}$ on $L_{a}^{2}\left(B_{n}\right)$. We need to show that $\varphi$ and $\psi$ satisfy one of the conditions in Theorem 2.2. By Proposition 6.1, there are four functions $f, g, h$, and $k$ in $H^{2 n}\left(S_{n}\right)$ such that

$$
\varphi=f+\bar{k} \text { and } \psi=h+\bar{g}
$$

Every function in $L^{p}\left(S_{n}\right)$ extends to an $\mathcal{M}$-harmonic function on the unit ball, via the Poisson integral. In fact, functions in the Hardy space correspond to holomorphic functions on the unit ball, and the evaluation at $z$ for any $z$ in $B_{n}$

$$
f \rightarrow f(z)
$$

is a bounded linear functional, so there is a function $S_{z}$ in $H^{2}\left(S_{n}\right)$ such that $f(z)=$ $\left\langle f, S_{z}\right\rangle$. It is easy to check that, for any function $u$ in $H^{2}\left(S_{n}\right)$,

$$
T_{\bar{u}}\left(S_{z}\right)=\overline{u(z)} S_{z}
$$


Then for $z \in B_{n}$,

$$
\begin{aligned}
& \left\langle T_{\psi} T_{\varphi} \frac{S_{z}}{\left\|S_{z}\right\|}, \frac{S_{z}}{\left\|S_{z}\right\|}\right\rangle=\left\langle T_{\varphi} \frac{S_{z}}{\left\|S_{z}\right\|}, T_{\bar{\psi}} \frac{S_{z}}{\left\|S_{z}\right\|}\right\rangle=\left\langle(f+\overline{k(z)}) \frac{S_{z}}{\left\|S_{z}\right\|}, \bar{\psi} \frac{S_{z}}{\left\|S_{z}\right\|}\right\rangle \\
& =\left\langle(\varphi+\overline{k(z)}-\bar{k}) \frac{S_{z}}{\left\|S_{z}\right\|}, \bar{\psi} \frac{S_{z}}{\left\|S_{z}\right\|}\right\rangle=\left\langle\varphi \frac{S_{z}}{\left\|S_{z}\right\|}, \bar{\psi} \frac{S_{z}}{\left\|S_{z}\right\|}\right\rangle+\left\langle(\overline{k(z)}-\bar{k}) \frac{S_{z}}{\left\|S_{z}\right\|}, \bar{\psi} \frac{S_{z}}{\left\|S_{z}\right\|}\right\rangle \\
& =\left\langle\psi \varphi \frac{S_{z}}{\left\|S_{z}\right\|}, \frac{S_{z}}{\left\|S_{z}\right\|}\right\rangle+\overline{k(z)} h(z)-\left\langle\bar{k} h \frac{S_{z}}{\left\|S_{z}\right\|}, \frac{S_{z}}{\left\|S_{z}\right\|}\right\rangle .
\end{aligned}
$$

Interchanging $\varphi$ and $\psi$ in the above equations gives

$$
\left\langle T_{\varphi} T_{\psi} \frac{S_{z}}{\left\|S_{z}\right\|}, \frac{S_{z}}{\left\|S_{z}\right\|}\right\rangle=\left\langle\varphi \psi \frac{S_{z}}{\left\|S_{z}\right\|}, \frac{S_{z}}{\left\|S_{z}\right\|}\right\rangle+\overline{g(z)} f(z)-\left\langle\bar{g} f \frac{S_{z}}{\left\|S_{z}\right\|}, \frac{S_{z}}{\left\|S_{z}\right\|}\right\rangle .
$$

Combining (6.17) with (6.18) gives

$$
\begin{aligned}
& \left\langle\left(T_{\varphi} T_{\psi}-T_{\psi} T_{\varphi}\right) \frac{S_{z}}{\left\|S_{z}\right\|}, \frac{S_{z}}{\left\|S_{z}\right\|}\right\rangle \\
& \quad=-\int_{S_{n}}(f(w) \overline{g(w)}-h(w) \overline{k(w)}) \frac{\left|S_{z}(w)\right|^{2}}{\left\|S_{z}\right\|^{2}} d \sigma(w)+(f(z) \overline{g(z)}-h(z) \overline{k(z)}) .
\end{aligned}
$$

Because $T_{\varphi}$ commutes with $T_{\psi}$, we have

$$
\int_{S_{n}}(f(w) \overline{g(w)}-h(w) \overline{k(w)}) \frac{\left|S_{z}(w)\right|^{2}}{\left\|S_{z}\right\|^{2}} d \sigma(w)=f(z) \overline{g(z)}-h(z) \overline{k(z)} .
$$

Thus the right hand side of the above equation defines an $\mathcal{M}$-harmonic function on the unit ball, and so the left hand side must be $\mathcal{M}$-harmonic on $B_{n}$. This implies that $f \bar{g}-h \bar{k}$ is $\mathcal{M}$-harmonic. By Theorem $5.6, f, g, h$, and $k$ satisfy one of the conditions in Theorem 5.6. It follows from Lemma 6.8 that $\varphi$ and $\psi$ satisfy one of the conditions of Theorem 2.2. This completes the proof.

\section{REFERENCES}

[AFR] P. Ahern, M. Flores and W. Rudin, An invariant volume-mean-value property, J. Functional Analysis 111 (1993), 380-397. MR 94b:31002

[AhR] P. Ahern and W. Rudin, M-harmonic products, Indag. Math. 2 (1991), 141-147. MR 92g:32013

[AxC] S. Axler and Z. Čučković, Commuting Toeplitz operators with harmonic symbols, Integral Equations Operator Theory 14 (1991), 1-12. MR 92f:47018

$[\mathrm{AxG}]$ S. Axler and P. Gorkin, Algebras on the disk and doubly commuting operators, Trans. Amer. Math. Soc. 309 (1988), 711-723. MR 90a:46133

[BrH] A. Brown and P.R. Halmos, Algebraic properties of Toeplitz operators, J. Reine Angew. Math. 213 (1964), 89-102. MR 28:3350

[CL] B. Choe and Y. Lee, Pluriharmonic symbols of commuting Toeplitz operators, Illinois J. Math. 37 (1993), 424-436. MR 94i:47041

[Eng] M. Engliš, Functions invariant under the Berezin transform, J. Functional Analysis 121 (1994), 233-254. MR 95h:31001

[Rud] W. Rudin, Function theory on the unit ball of $C^{n}$, Grundlehren 241, Springer-Verlag, Berlin-Heidelberg-New York, 1980. MR 82i:32002 
[Zh1] D. Zheng, Hankel operators and Toeplitz operator on the Bergman space, J. Functional Analysis 83 (1989), 98-120. MR 91b:47057

[Zh2] D. Zheng, Semi-commutators of Toeplitz operators on the Bergman space, Integral Equations Operator Theory 25 (1996), 347-372. MR 97e:47039

Department of Mathematics, Michigan State University, East Lansing, Michigan 48824

Current address: Department of Mathematics, Vanderbilt University, Nashville, Tennessee 37240

E-mail address: zheng@math.vanderbilt.edu 